\title{
Validade e clareza dos conceitos e terminologias do Guia de Atividade Física para a População Brasileira
}

\section{Validity and Clarity of the Concepts and Terminologies of the Physical Activity Guidelines for the Brazilian Population}

\section{AUTORES \\ Tânia Rosane Bertoldo Benedetti ${ }^{1}$ (D) \\ Lucélia Justino Borges ${ }^{2}$ (D) \\ Inês Amanda Streit ${ }^{3}$ (ID \\ Leandro Martin Totaro Garcia ${ }^{4}$ (D) \\ Sofia Wolker Manta ${ }^{5}$ (D) \\ Gerfeson Mendonça ${ }^{6}$ (iD) \\ Maria Angélica Binotto 7 (D) \\ Marina Christofoletti ${ }^{1}$ (ID \\ Fernando Lopes e Silva-Júnior ${ }^{8}$ (D) \\ Pedro Curi Hallal ${ }^{9}$ (D) \\ Camila Bosquiero Papini ${ }^{10}$ (iD \\ 1 Universidade Federal de Santa Catarina, \\ Departamento de Educacão Física, Florianópolis, \\ Santa Catarina, Brasil. \\ 2 Universidade Federal do Paraná, Departamento de Educação Física, Curitiba, Paraná, Brasil \\ 3 Universidade Federal do Amazonas, Manaus, Amazonas, Brasil. \\ 4 Queen's University Belfast, Centre for Public Health, Belfast, Reino Unido. \\ 5 Ministério da Saúde, Secretaria de Atenção \\ Primária à Saúde, Departamento de Promoção \\ da Saúde, Coordenaçâao-Geral de Promoção da \\ Atividade Física e Ações Intersetoriais, Brasília, \\ Distrito Federal, Brasil. \\ 6 Universidade Federal de Alagoas e Centro Universitário CESMAC, Maceió, Alagoas, Brasil. \\ 7 Universidade Estadual do Centro-Oeste, \\ Departamento de Educação Física, Irati, Paraná, Brasil. \\ 8 Universidade Federal do Piauí/Universidade \\ Federal do Delta do Parnaíba, Coordenação do \\ Curso de Medicina, Parnaíba, Piauí, Brasil. \\ 9 Universidade Federal de Pelotas, Departamento \\ de Ginástica e Saúde, Pelotas, Rio Grande do Sul, \\ Brasil. \\ 10 Universidade Federal do Triângulo Mineiro, \\ Departamento de Ciências do Esporte, Uberaba, \\ Minas Gerais, Brasil.}

\section{CONTATO}

Tânia Rosane Bertoldo Benedetti

tania.benedetti@ufsc.br

Campus Universitário Reitor João David

Ferreira Lima, Florianópolis, Santa Catarina,

Brasil.

CEP: 88040-900.

\section{DOI}

$10.12820 /$ rbafs.26e0212

\begin{abstract}
RESUMO
Nosso objetivo foi avaliar a validade e a clareza dos conceitos e terminologias adotados na elaboração do Guia de Atividade Física para a População Brasileira (Guia). O Grupo de Trabalho Domínios da Atividade Física (GT Domínios) conduziu a avaliação da validade e da clareza dos conceitos e terminologias relacionados a atividade física (AF), comportamento sedentário, domínios (tempo livre, deslocamento, trabalho ou estudo e tarefas domésticas) e intensidades da AF (leve, moderada e vigorosa), em três etapas: 1- Proposição dos conceitos; 2- Escuta com pesquisadores (dois momentos); 3- Consulta pública. Os conceitos propostos pelo GT Domínios foram baseados em guias internacionais, artigos científicos, relatórios nacionais e conhecimentos técnico-científicos-acadêmicos dos integrantes do GT Domínios, discutidos em reuniões (etapa 1). Na escuta com pesquisadores (etapa 2) foram testadas a validade e a clareza dos conceitos em dois momentos. Participaram 70 e 40 pesquisadores vinculados aos outros GT do Guia no primeiro e segundo momentos, respectivamente. Em ambas as escutas, todos os conceitos apresentaram índice de concordância para validade e clareza igual ou superior a $80 \%$. As sugestões convergentes indicadas na etapa 2 foram incluídas e novas versões dos conceitos foram disponibilizadas para a terceira etapa (consulta pública) realizada pelo Ministério da Saúde. Foram realizadas 14 sugestões relacionadas aos conceitos que foram aceitas e incorporadas ao texto do Guia, quando pertinentes. Conclui-se que os conceitos e terminologias relacionados a AF propostos pelo GT Domínios para compor o Guia, após a avaliação de pesquisadores especialistas e da população consultada, são válidos e claros.
\end{abstract}

Palavras-chave: Atividade motora; Terminologia; Brasil, Promoção da Saúde.

ABSTRACT

We evaluated the validity and clarity of the concepts and terminologies adopted to develop the Physical Activity Guidelines for the Brazilian Population (Guidelines). The Physical Activity Domains Working Group (Domains $W G$ ) assessed the validity and clarity of the following concepts: physical activity, sedentary behavior, PA domains (free time, commuting, work or study, and domestic tasks) and intensities (light, moderate and vigorous). The concepts were evaluated in three stages: 1- Concept proposition; 2- Expert consultation (two steps); 3- Public consultation. The concepts proposed by the Domains WG were based on international guidelines, scientific articles, official documents from the Ministry of Health, and technical-scientific-academic knowledge of the Domains WG members, discussed in a series of meetings (stage 1). In the second stage (expert consultation), validity and clarity of the concepts were tested on two occasions. Seventy and forty researchers working in the other Guidelines WGs contributed to first and second steps, respectively. In both occasions, validity and clarity agreement were equal to or greater than $80 \%$ for all concepts. Suggestions provided in step 2 were adopted, and new versions of the concepts were made available for the third step, a public consultation carried out by the Ministry of Health. Fourteen suggestions related to the concepts were made and, when relevant, included in the Guidelines. According to the assessment and suggestions by experts and members of the public, the concepts and terminologies proposed by the Domains $W G$ for the Guidelines are valid and clear.

Keywords: Motor activity; Terminology; Brazil; Health Promotion.

\section{(cc) BY}

Este trabalho está licenciado com uma Licença Creative Commons - Atribuição 4.0 Internacional. 


\section{Introdução}

A prática regular de atividade física $(\mathrm{AF})$ é fator de proteção para o controle das doenças crônicas não transmissíveis, contribui para o bem-estar geral e para a saúde mental ${ }^{1}$. Entretanto, $44,8 \%$ dos adultos brasileiros não atendem às recomendações da prática de AF preconizadas pela Organização Mundial da Saúde $(\mathrm{OMS})^{2}$. O monitoramento da prática de $\mathrm{AF}$ em adultos brasileiros também revela diferenças entre sexo, idade e escolaridade ${ }^{2}$.

A inexistência de recomendações para a prática de AF específicas para a população brasileira motivou a elaboração de um guia com recomendações à população. Buscando preencher parte desta lacuna foi elaborado, em 2020, o primeiro Guia de Atividade Física para a População Brasileira (Guia), que teve seu lançamento em 2021.

O Guia tem como objetivo oferecer orientações, incentivar e facilitar a adoção da prática de AF no cotidiano da população. Suas recomendações buscam auxiliar a tomada de decisões dos diferentes atores e setores envolvidos na promoção da saúde, fornecendo suporte para as políticas públicas que visem o aumento dos níveis da AF da população brasileira. Para tanto, torna-se de grande importância que os conceitos e terminologias norteadores do Guia possam ao mesmo tempo refletir o conhecimento técnico-científico-acadêmico para profissionais, gestores da saúde e outros setores, e também transmitir uma mensagem válida e clara para a população em geral.

Assim, validar os conceitos e as terminologias do Guia facilita o entendimento e a delimitação dos fenômenos, além de esclarecer o que se quer expressar, deixando claro o que se pretende dizer nas diferentes formas com que são utilizados. Ao mesmo tempo, os conceitos e as terminologias podem apresentar diferentes definições, como se tem observado na área da AF e saúde.

Desse modo, é fundamental apresentar estes termos e conceitos traduzidos para o cotidiano e o contexto profissional da população brasileira. Portanto, nosso objetivo foi avaliar a validade e a clareza dos conceitos e terminologias adotados na elaboração do Guia.

\section{Métodos}

Para a elaboração do Guia, inicialmente foi constituído um Comitê Científico (CC), coordenado pela Universidade Federal de Pelotas, que organizou e planejou a estrutura do Guia contendo oito Grupos de Trabalhos (GTs). A Fundação Delfim Mendes Silveira estabeleceu critérios e lançou dois editais de seleção para com- posição de equipe do Guia, sendo um edital para oito vagas de coordenadores dos GTs ( ${ }^{\circ} 05$ de 13 de março de 2020) e outro para 54 pesquisadores bolsistas ( ${ }^{\circ} 06$ de 19 de março de 2020). Em cada GT, atuaram um representante do CC, um representante do Ministério da Saúde (MS), um coordenador e sete membros. Todos os membros dos GTs eram pesquisadores com título de doutor e/ou mestre. Dentre os GTs criados, o primeiro se refere aos Domínios da Atividade Física (GT Domínios), que teve como objetivo conduzir a proposição e avaliação da validade e da clareza dos conceitos e terminologias a serem utilizados no Guia.

\section{Planejamento e organização do trabalho do GT Domínios}

O GT Domínios foi composto por dez pesquisadores (seis mulheres e quatro homens) de diferentes regiões do Brasil (Sul, Sudeste, Norte e Nordeste), com formação em Educação Física (licenciatura e/ou bacharelado) e experiência e atuação profissional na área da $\mathrm{AF}$ relacionada à saúde. $\mathrm{O}$ grupo se reuniu semanalmente de forma remota no período de maio a agosto no ano de 2020. O GT Domínios elaborou a proposição dos conceitos e termos de AF, comportamento sedentário (CS), domínios da AF (tempo livre, deslocamento, trabalho ou estudo, e tarefas domésticas) e intensidades da AF (leve, moderada e vigorosa). Essa elaboração foi conduzida considerando procedimentos de validade e clareza, com o foco em adequar para a população, com linguagem clara e acessível. Dessa forma, foram realizados procedimentos em três etapas: 1) Proposição dos conceitos pelo GT Domínios; 2) Escuta com pesquisadores dos demais GTs do Guia; 3) Consulta Pública.

\section{Proposição dos conceitos pelo GT Domínios}

Os conceitos iniciais de AF, CS, domínios da AF (tempo livre, deslocamento, trabalho ou estudo e tarefas domésticas) e intensidades da AF (leve, moderada e vigorosa) propostos pelo GT Domínios foram baseados em guias internacionais ${ }^{1,2,4-8}$, artigos científicos ${ }^{1,9-12}$, relatórios de pesquisa do $\mathrm{MS}^{2}$ e do Programa das $\mathrm{Na}^{-}$ ções Unidas para o Desenvolvimento (PNUD) ${ }^{13}$, e nos conhecimentos técnico-científicos dos pesquisadores do GT, e foram discutidos em reuniões periódicas.

Escuta com pesquisadores dos demais GT do Guia Essa etapa foi caracterizada por escutas com os pesquisadores dos demais GTs para avaliar a validade e a clareza dos conceitos a serem adotados pelo Guia. Foi 
utilizado o processo de validação conforme apontado por Santos et al. ${ }^{15}$. Considerou-se que, para um instrumento ter validade e clareza, os índices devem atingir pelo menos $80 \%{ }^{16}$.

A escuta foi realizada em dois momentos distintos, por meio de matrizes analíticas organizadas em dois formulários no Google ${ }^{\circledR}$ Forms (links disponíveis em: https://forms.gle/263GGE4Nv1qRa4jh6 e https:// forms.gle/m83rYKqNqCN5B6nX8).

A Matriz Analítica 1, aplicada de 24 junho a 7 de julho de 2020, continha 70 questões para avaliação dos conceitos de AF, domínios da AF (tempo livre, deslocamento, trabalho ou estudo, e tarefas domésticas) e intensidades da AF (leve, moderada e vigorosa). A Matriz Analítica 2, aplicada de 21 a 25 de agosto de 2020, continha 13 questões para avaliação do conceito de CS. Em ambas as matrizes, cada conceito foi avaliado com as seguintes informações: a) Escala Likert de 1 a 4 para validade (1 não válido e 4 válido) e clareza (1 não claro e 4 claro); b) Sugestões para melhorar os conceitos e os exemplos quanto à validade e à clareza; c) Campo específico para acréscimo de outros comentários. Participaram da primeira escuta 70 pesquisadores e da segunda 40 pesquisadores vinculados aos outros sete GTs. Todos os participantes da escuta assinaram a Declaração de Confidencialidade e Conflito de Interesses disponibilizada pelo Departamento de Promoção da Saúde do Ministério da Saúde.

A coleta de dados ocorreu de forma semelhante em ambos os momentos da escuta. Todos os integrantes dos demais GTs foram convidados a participar da escuta. O coordenador de cada GT recebeu o link do formulário on-line e encaminhou o convite aos demais integrantes de seu GT.

\section{Tratamento dos dados da escuta}

Os dados quantitativos foram tabulados no programa Microsoft Office Excel e analisados por estatística descritiva (média, desvio-padrão e porcentagem).

Para os dados qualitativos, as respostas de cada conceito foram organizadas e sistematizadas em quadros. As sugestões semelhantes foram agrupadas considerando as alterações que envolveram inclusão, exclusão ou substituição de termos ou dos exemplos ${ }^{16}$. Em seguida, os resultados foram analisados e discutidos pelo GT Domínios, sendo que a reformulação dos conceitos incluiu os pontos convergentes, pautando-se no referencial teórico adotado e na experiência profissional dos integrantes do GT. Os conceitos reformulados na etapa 2 foram enviados para análise da consulta pública (etapa 3).

\section{Consulta Pública}

Nessa etapa, o capítulo produzido contendo os conceitos desenvolvidos pelo GT Domínios foi enviado ao MS e postado para consulta pública, com o restante do Guia, no período de 14 a 31 de agosto de 2020. A consulta pública teve o acesso amplamente divulgado e as contribuições foram realizadas por meio de um formulário on-line específico, elaborado pelo Departamento de Promoção da Saúde do MS.

Participaram desta consulta membros de diversos setores da sociedade, como estudantes e profissionais de diferentes áreas do conhecimento, gestores de secretarias, professores da educação básica e superior, profissionais da Atenção Básica e organizações sociais, entre outros. Foram realizadas 265 sugestões para o Guia, sendo 56 sugestões específicas para todo o conteúdo do capítulo produzido pelo GT Domínios, embora algumas repetidas.

\section{Aspectos éticos}

As atividades de escuta com os atores relacionados ao Guia foram utilizadas com objetivo de incorporar diferentes perspectivas no conteúdo do documento. A abordagem derivou-se de modelos já existentes no qual diversos atores (profissionais, gestores, usuários de serviços de saúde, representantes de entidades, etc.) são envolvidos no processo de desenvolvimento de documentos voltados à saúde pública. Portanto, ao longo da elaboração do Guia, não consideramos tais indivíduos como "participantes de pesquisa", bem como não coletamos informações com fins de investigação nestes indivíduos. Pelo contrário, consideramos os participantes em grupos de escuta como colaboradores na concepção de conteúdos e teor do Guia.

Assim, foram seguidas disposições de órgãos internacionais Agência Nacional de Segurança do Paciente (National Health System, Reino Unido), INVOLVE (ONG para envolvimento público em tomada de decisão no Reino Unido) ${ }^{17}$, e o MS por meio de sua Resolução do Conselho Nacional de Saúde N0 510 de 07 de abril de 2016, artigo $1^{0}$, parágrafo único, o qual aborda que "Não serão registradas nem avaliadas pelo sistema CEP/CONEP: I - pesquisa de opinião pública com participantes não identificados; VI - pesquisa realizada exclusivamente com textos científicos para revisão da literatura científica; e VII - pesquisa que objetiva o 
aprofundamento teórico de situações que emergem espontânea e contingencialmente na prática profissional, desde que não revelem dados que possam identificar o sujeito" ${ }^{18}$.

\section{Resultados}

Os resultados da análise da validade, da clareza e do índice de concordância dos conceitos e terminologias inicialmente propostos pelo GT Domínios estão apresentados na Tabela 1 . Os valores variaram de 3,4 a 3,8 para a validade e de 3,3 a 3,7 para a clareza. Todos os conceitos e terminologias apresentaram índice de concordância igual ou superior a $80 \%$.

Tabela 1 - Média, desvio-padrão e índice de concordância da validade e clareza dos conceitos elaborados para o Guia de Atividade Física para a População Brasileira (Escala Likert de 4 pontos) 2020.

\begin{tabular}{lcccc}
\hline Conceito & Validade & $\begin{array}{c}\text { Índice de } \\
\text { concordância }\end{array}$ & Clareza & $\begin{array}{c}\text { Índice de } \\
\text { concordância }\end{array}$ \\
\hline AF & $3,7 \pm 0,6$ & $92,5 \%$ & $3,6 \pm 0,6$ & $90,0 \%$ \\
Domínios da AF & $3,6 \pm 0,7$ & $90,0 \%$ & $3,5 \pm 0,7$ & $87,5 \%$ \\
AF no trabalho ou & $3,4 \pm 0,8$ & $85,0 \%$ & $3,4 \pm 0,8$ & $85,0 \%$ \\
estudo & $3,6 \pm 0,6$ & $90,0 \%$ & $3,7 \pm 0,6$ & $92,5 \%$ \\
AF no deslocamento & $3,6 \pm 0,5,7 \pm 0,5$ & $92,5 \%$ \\
AF nas tarefas & $3,8 \pm 0,5$ & $95,0 \%$ & 3,7 \\
domésticas & $3,4 \pm 0,8$ & $85,0 \%$ & $3,5 \pm 0,7$ & $87,5 \%$ \\
AF no tempo livre & $3,6 \pm 0,8$ & $90,0 \%$ & $3,2 \pm 0,9$ & $80,0 \%$ \\
Intensidade & $3,7 \pm 0,7$ & $92,5 \%$ & $3,3 \pm 0,8$ & $82,5 \%$ \\
AF leve & $3,6 \pm 0,7$ & $90,0 \%$ & $3,3 \pm 0,9$ & $82,5 \%$ \\
AF moderada & $3,6 \pm 0,7$ & $90,0 \%$ & $3,3 \pm 0,8$ & $82,5 \%$ \\
AF vigorosa & $3,8 \pm 0,5$ & $95,0 \%$ & $3,5 \pm 0,8$ & $87,5 \%$ \\
$\begin{array}{l}\text { Comportamento } \\
\text { sedentário }\end{array}$ & &
\end{tabular}

$\mathrm{AF}=$ atividade física

\section{Desenvolvimento dos conceitos}

Os conceitos e as terminologias estão apresentados nos Quadros 1 a 3, considerando a proposta inicial desenvolvida pelo GT Domínios, a versão alterada após a escuta com os pesquisadores dos demais GT e enviada ao MS, e os conceitos e terminologias finais incorporando as sugestões da consulta pública.

Após a consulta pública, 14 sugestões foram relacionadas especificamente aos conceitos e terminologias propostos pelo GT Domínios, sendo que a maioria não levou a mudanças significativas no conteúdo do texto. As sugestões foram para inclusão ou exclusão de exemplos $(n=6)$, relacionadas à alteração de palavras na frase $(n=4)$, sugestões de reescrita de frases sem alteração do conteúdo $(\mathrm{n}=2)$, críticas em relação à "dosagem" da intensidade $(n=1)$. Somente uma sugestão foi relacionada à mudança do conceito de CS, mas não foi aceita por não atender o escopo e a proposta inicial do conceito.

Os Quadros 1, 2 e 3 apresentam a proposição dos conceitos e terminologias, bem como os exemplos em cada uma das etapas de avaliação para AF e CS, domínios e intensidade da $\mathrm{AF}$, respectivamente.

A Figura 1 apresenta uma síntese dos conceitos e terminologias desenvolvidos para o Guia.

Com relação aos domínios da AF (Quadro 2), considerando a proposição dos conceitos e terminologias com linguagem clara e acessível à população, os resultados da escuta mostraram que $87 \%$ dos pesquisadores indicaram "deslocamento" e 13\% "transporte" para o nome do domínio. Em relação ao domínio do tempo livre, 56,5\% dos participantes da escuta sugeriram que a definição do domínio deveria envolver as atividades realizadas no tempo livre, enquanto 43,5\% sugeriram que a definição deveria incluir atividades realizadas no lazer.

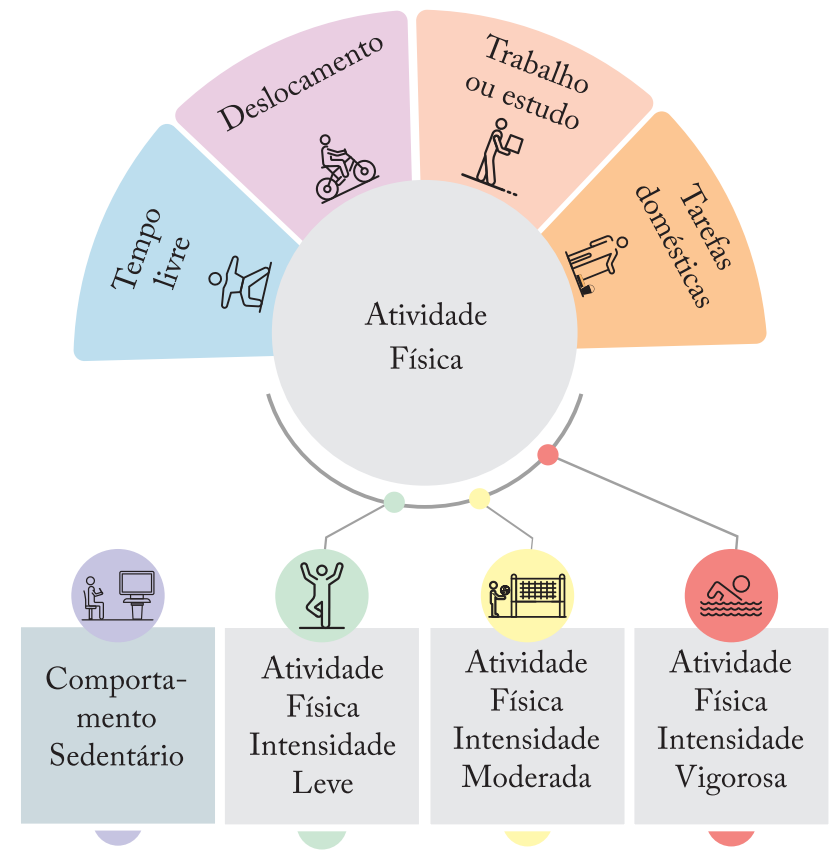

Figura 1 - Conceitos e terminologias do Guia de Atividade Física para a População Brasileira 2020.

\section{Discussão}

No presente estudo apresentamos o processo de avaliação da validade e clareza dos conceitos e terminologias adotados pelo Guia, contribuindo dessa maneira para a educação para o incentivo e adoção da prática de AF pela população brasileira. 
Quadro 1 - Processo de elaboração dos conceitos e terminologias de Atividade Física e Comportamento Sedentário, para o Guia de Atividade Física para a População Brasileira 2020.

\begin{tabular}{|c|c|c|c|}
\hline Termos & $\begin{array}{l}\text { Proposição inicial do conceito } \\
\text { (Etapa 1) }\end{array}$ & $\begin{array}{l}\text { Proposição do conceito após escuta aos GT } \\
\text { (Etapa 2) }\end{array}$ & $\begin{array}{l}\text { Conceito final definido após a consulta } \\
\text { pública } \\
\text { (Etapa 3) }\end{array}$ \\
\hline Atividade física & $\begin{array}{l}\text { A atividade física é um comportamento } \\
\text { com distintos significados, que envolve os } \\
\text { movimentos voluntários do corpo, com gasto } \\
\text { energético acima dos níveis de repouso, } \\
\text { que oportuniza interações sociais e com } \\
\text { o ambiente, podendo ocorrer no lazer, no } \\
\text { deslocamento, nas tarefas domésticas e no } \\
\text { trabalho/educacional. } \\
\text { São exemplos de atividades físicas: caminhar, } \\
\text { correr, subir escadas, carregar, empurrar, } \\
\text { dançar, praticar lutas, limpar a casa, praticar } \\
\text { yoga, pedalar, praticar esportes, brincar, } \\
\text { engatinhar, passear com o cachorro, cultivar a } \\
\text { terra, praticar ginástica, cuidar do quintal... }\end{array}$ & $\begin{array}{l}\text { Atividade física é um comportamento que } \\
\text { envolve os movimentos voluntários do corpo, } \\
\text { com gasto energético acima dos níveis de } \\
\text { repouso, que oportuniza interações sociais e } \\
\text { com o ambiente, podendo acontecer no lazer, } \\
\text { no deslocamento, nas tarefas domésticas e no } \\
\text { trabalho/estudo. } \\
\text { São exemplos de atividade física: caminhar, } \\
\text { correr, pedalar, brincar, subir escadas, carregar, } \\
\text { dançar, praticar esportes, praticar lutas, } \\
\text { praticar ginásticas, praticar yoga, praticar } \\
\text { liang gong, praticar tai chi chuan, limpar a } \\
\text { casa, engatinhar, passear com o cachorro, } \\
\text { cultivar a terra, cuidar do quintal... }\end{array}$ & $\begin{array}{l}\text { Atividade física é um comportamento que } \\
\text { envolve os movimentos voluntários do } \\
\text { corpo, com gasto de energia acima do nível } \\
\text { de repouso, promovendo interações sociais } \\
\text { e com o ambiente, podendo acontecer no } \\
\text { tempo livre, no deslocamento, no trabalho ou } \\
\text { estudo e nas tarefas domésticas. } \\
\text { São exemplos de atividade física: caminhar, } \\
\text { correr, pedalar, brincar, subir escadas, carregar } \\
\text { objetos, dançar, limpar a casa, passear com } \\
\text { animais de estimação, cultivar a terra, cuidar } \\
\text { do quintal, praticar yoga, liang gong, esportes, } \\
\text { lutas, ginásticas, entre outros. }\end{array}$ \\
\hline $\begin{array}{l}\text { Comportamento } \\
\text { sedentário }\end{array}$ & $\begin{array}{l}\text { Comportamento sedentário é qualquer } \\
\text { atividade realizada, na posição sentada, } \\
\text { reclinada ou deitada quando se está acordado } \\
\text { e gastando pouca energia. Ou seja, quando } \\
\text { você está sentado ou deitado assistindo } \\
\text { televisão, mexendo no celular, trabalhando no } \\
\text { computador, assistindo aula ou no trânsito } \\
\text { dentro do carro ou ônibus. }\end{array}$ & $\begin{array}{l}\text { Comportamento sedentário é qualquer } \\
\text { atividade realizada na posição sentada, } \\
\text { reclinada ou deitada, quando você está } \\
\text { acordado e gastando pouca energia. Como } \\
\text { exemplo, quando você está sentado, reclinado } \\
\text { ou deitado para usar dispositivos eletrônicos } \\
\text { (televisão, videogames, computador, tablet } \\
\text { e celular), assistir aula, realizar trabalhos } \\
\text { manuais, jogar cartas ou jogos de mesa ou } \\
\text { dentro do carro, ônibus ou metrô. }\end{array}$ & $\begin{array}{l}\text { Comportamento sedentário são atividades } \\
\text { realizadas quando você está acordado } \\
\text { sentado, reclinado ou deitado e gastando } \\
\text { pouca energia. Por exemplo, quando você } \\
\text { está em uma dessas posições para usar celular, } \\
\text { computador, tablet, videogame e } \\
\text { assistir televisão ou aula, realizar trabalhos } \\
\text { manuais, jogar cartas ou jogos de mesa, } \\
\text { dentro do carro, ônibus ou metrô. }\end{array}$ \\
\hline
\end{tabular}

Quadro 2 - Processo de elaboração dos conceitos e terminologias de Domínios da Atividade Física, para o Guia de Atividade Física para a População Brasileira 2020.

\begin{tabular}{|c|c|c|c|}
\hline Termos & $\begin{array}{l}\text { Proposição inicial do conceito } \\
\text { (Etapa 1) }\end{array}$ & $\begin{array}{l}\text { Proposição do conceito definido após escuta } \\
\text { aos GT } \\
(\text { Etapa 2) }\end{array}$ & $\begin{array}{l}\text { Conceito final definido após a consulta } \\
\text { pública } \\
\text { (Etapa 3) }\end{array}$ \\
\hline Domínios & $\begin{array}{l}\text { São os momentos e as situações em } \\
\text { que as atividades físicas são acumuladas } \\
\text { durante o dia, podendo ser no trabalho, no } \\
\text { deslocamento, nas tarefas domésticas e no } \\
\text { lazer. }\end{array}$ & $\begin{array}{l}\text { Os domínios representam o propósito } \\
\text { da atividade física, podendo ser realizada } \\
\text { no lazer, no deslocamento, nas tarefas } \\
\text { domésticas e no trabalho/educacional. }\end{array}$ & O conceito "domínios" foi suprimido. \\
\hline $\begin{array}{l}\text { Domínio do } \\
\text { tempo livre }\end{array}$ & $\begin{array}{l}\text { la } \\
\text { São aquelas realizadas no lazer baseadas em } \\
\text { preferências e oportunidades. } \\
\text { São atividades físicas no lazer: nadar; } \\
\text { praticar yoga, ginástica, musculação, tai } \\
\text { chi chuan, caminhada, judô; fazer trilha; } \\
\text { dançar; surfar; jogar peteca, futebol, capoeira, } \\
\text { frescobol, voleibol. } \\
\text { Obs: A proposta inicial do GT para o nome } \\
\text { deste domínio foi lazer. }\end{array}$ & $\begin{array}{l}\text { São atividades realizadas no tempo livre } \\
\text { e no lazer baseadas em preferências e } \\
\text { oportunidades. } \\
\text { São exemplos de atividade física no lazer: } \\
\text { caminhar, correr, empinar pipa, dançar, } \\
\text { nadar, brincar, fazer trilha, pedalar, surfar, } \\
\text { pular corda, praticar ginásticas, musculação, } \\
\text { hidroginástica, artes marciais, yoga, liang } \\
\text { gong, tai chi chuan, jogar futebol, voleibol, } \\
\text { basquetebol, bocha, tênis, peteca e frescobol, } \\
\text { que podem ser praticadas na residência } \\
\text { (casa, apartamento e condomínio), em } \\
\text { espaços públicos (praças, ruas, parques, } \\
\text { praias, quadras, centros esportivos e de } \\
\text { lazer, Academias da Saúde, Unidades de } \\
\text { Saúde, ciclovias, centros comunitários,...) } \\
\text { e privados (academias, clubes, estúdios, } \\
\text { associações esportivas, quadras, ...). } \\
\text { Obs: Após a escuta, a proposta para o nome } \\
\text { deste domínio permaneceu lazer. }\end{array}$ & $\begin{array}{l}\text { A atividade física no tempo livre é feita no } \\
\text { seu tempo disponível ou no lazer, baseada } \\
\text { em preferências e oportunidades. Você pode } \\
\text { caminhar; correr; empinar pipa; dançar; } \\
\text { nadar; fazer trilha; pedalar; surfar; pular } \\
\text { corda; jogar futebol, vôlei, basquete, bocha, } \\
\text { tênis, peteca, taco/bete ou frescobol; praticar } \\
\text { ginástica, musculação, hidroginástica, artes } \\
\text { marciais, capoeira, yoga, ou participar de } \\
\text { brincadeiras e jogos, como esconde-esconde, } \\
\text { pega-pega, pular corda, saltar elástico, } \\
\text { queimada/baleado/carimba/caçador, entre } \\
\text { outras. }\end{array}$ \\
\hline
\end{tabular}


Continuação do Quadro 2 - Processo de elaboração dos conceitos e terminologias de Domínios da Atividade Física, para o Guia de Atividade Física para a População Brasileira 2020.

\begin{tabular}{|c|c|c|c|}
\hline Termos & $\begin{array}{l}\text { Proposição inicial do conceito } \\
\text { (Etapa 1) }\end{array}$ & $\begin{array}{l}\text { Proposição do conceito definido após escuta } \\
\text { aos GT } \\
\text { (Etapa 2) }\end{array}$ & $\begin{array}{l}\text { Conceito final definido após a consulta } \\
\text { pública } \\
\text { (Etapa 3) }\end{array}$ \\
\hline $\begin{array}{l}\text { Domínio do } \\
\text { deslocamento }\end{array}$ & $\begin{array}{l}\text { São aquelas realizadas como forma de } \\
\text { deslocamento ativo de um lugar para o } \\
\text { outro. } \\
\text { São atividades físicas no deslocamento: } \\
\text { caminhar, manejar cadeira de rodas, remar } \\
\text { canoa, andar de bicicleta, skate, patins. }\end{array}$ & $\begin{array}{l}\text { São atividades realizadas como forma de } \\
\text { deslocamento ativo para ir de um lugar a } \\
\text { outro. } \\
\text { São exemplos de atividade física no } \\
\text { deslocamento: caminhar, manejar cadeira de } \\
\text { rodas, pedalar, remar, patinar, andar à cavalo } \\
\text { e de skate para ir ao trabalho, à escola, aos } \\
\text { pontos de ônibus, às estações de metrô/ } \\
\text { trem, aos supermercados, às padarias, aos } \\
\text { restaurantes, aos bancos, à universidade, aos } \\
\text { estabelecimentos comerciais, aos templos } \\
\text { religiosos, para ir visitar amigos, familiares... }\end{array}$ & $\begin{array}{l}\text { A atividade física no deslocamento é feita } \\
\text { como forma de deslocamento ativo para ir } \\
\text { de um lugar a outro. Você pode caminhar, } \\
\text { manejar a cadeira de rodas, pedalar, remar, } \\
\text { patinar, andar a cavalo, de skate ou de } \\
\text { patinete (sem motor), entre outras. }\end{array}$ \\
\hline $\begin{array}{l}\text { Domínio do } \\
\text { trabalho ou } \\
\text { estudo }\end{array}$ & $\begin{array}{l}\text { São aquelas realizadas na jornada de trabalho } \\
\text { remunerado ou voluntário. } \\
\text { São atividades físicas no trabalho: carregar } \\
\text { peso, pescar, capinar, cozinhar. }\end{array}$ & $\begin{array}{l}\text { São atividades realizadas no trabalho } \\
\text { (remunerado ou não) e no contexto } \\
\text { educacional para desempenhar a função de } \\
\text { trabalho ou estudo. } \\
\text { São exemplos de atividades físicas no } \\
\text { trabalho: plantar, capinar, colher, caminhar, } \\
\text { correr, pedalar, limpar, varrer, lavar, ordenhar, } \\
\text { carregar peso que podem ser praticadas no } \\
\text { escritório, na indústria, na construção civil, } \\
\text { na oficina, na agricultura, na pecuária, na } \\
\text { piscicultura, na sala de aula, nas aulas de } \\
\text { educação física, nos esportes profissionais. }\end{array}$ & $\begin{array}{l}\text { A atividade física no trabalho ou estudo } \\
\text { é feita no trabalho e em atividades } \\
\text { educacionais, para desempenhar suas funções } \\
\text { laborais ou de estudo. Você pode plantar, } \\
\text { capinar, colher, caminhar, correr, pedalar, } \\
\text { limpar, varrer, lavar, ordenhar, carregar } \\
\text { objetos, participar das aulas de educação } \\
\text { física, brincar no recreio ou intervalo entre } \\
\text { as aulas e também antes ou depois das aulas, } \\
\text { entre outras. }\end{array}$ \\
\hline $\begin{array}{l}\text { Domínio das } \\
\text { tarefas domésticas }\end{array}$ & $\begin{array}{l}\text { São aquelas realizadas nas tarefas domésticas } \\
\text { para o cuidado do lar e da família. } \\
\text { São atividades físicas nas tarefas domésticas: } \\
\text { esfregar o chão, fazer feira, lavar roupa, varrer } \\
\text { a calçada, regar as plantas, fazer faxina, cortar } \\
\text { a grama. }\end{array}$ & $\begin{array}{l}\text { São atividades realizadas nas tarefas } \\
\text { domésticas para o cuidado do lar e da } \\
\text { família. } \\
\text { São exemplos de atividade física nas tarefas } \\
\text { domésticas: varrer, esfregar, lavar o chão, } \\
\text { a parede, os vidros; fazer compras; cortar } \\
\text { a grama, cuidar das plantas; dar banho na } \\
\text { criança, na pessoa que requer cuidados, no } \\
\text { cachorro. }\end{array}$ & $\begin{array}{l}\text { A atividade física nas tarefas domésticas é } \\
\text { feita para o cuidado do lar e da família. Você } \\
\text { pode cuidar das plantas, fazer compras, dar } \\
\text { banho na criança, no idoso, na pessoa que } \\
\text { requer cuidados ou no animal de estimação, } \\
\text { varrer, esfregar ou lavar, entre outras. }\end{array}$ \\
\hline
\end{tabular}

Quadro 3 - Processo de elaboração dos conceitos e terminologias das Intensidades da Atividade Física, para o Guia de Atividade Física para a População Brasileira 2020.

\begin{tabular}{|c|c|c|c|}
\hline Termos & $\begin{array}{l}\text { Proposição inicial do conceito } \\
\text { (Etapa 1) }\end{array}$ & $\begin{array}{l}\text { Proposição do conceito definido após } \\
\text { escuta aos GT } \\
\text { (Etapa 2) }\end{array}$ & $\begin{array}{l}\text { Conceito final definido após a consulta } \\
\text { pública } \\
\text { (Etapa 3) }\end{array}$ \\
\hline Intensidades & $\begin{array}{l}\text { Intensidade é a magnitude do esforço } \\
\text { necessário para realizar uma atividade } \\
\text { física, expressa pela variação dos batimentos } \\
\text { cardíacos, da frequência respiratória e da taxa } \\
\text { do gasto energético. }\end{array}$ & $\begin{array}{l}\text { Intensidade é o grau do esforço físico } \\
\text { necessário para realizar uma atividade } \\
\text { física. Normalmente quanto maior a } \\
\text { intensidade, há aumento dos batimentos } \\
\text { cardíacos, da frequência respiratória, } \\
\text { do gasto energético e da percepção do } \\
\text { esforço. }\end{array}$ & $\begin{array}{l}\text { A intensidade é o grau do esforço físico } \\
\text { necessário para fazer uma atividade física. } \\
\text { Normalmente, quanto maior a intensidade, } \\
\text { maior é o aumento dos batimentos do } \\
\text { coração, da respiração, do gasto de energia e } \\
\text { da percepção de esforço. }\end{array}$ \\
\hline Intensidade leve & $\begin{array}{l}\text { São aquelas que causam pequeno aumento } \\
\text { na respiração e nos batimentos cardíacos. } \\
\text { O gasto energético é entre } 1,6 \text { e } 2,9 \\
\text { equivalentes metabólicos. A percepção } \\
\text { individual de esforço está entre } 1 \text { e } 4 \text {, em } \\
\text { uma escala de zero a } 10 \text {. Nas atividades } \\
\text { físicas leves, consegue-se respirar } \\
\text { tranquilamente e conversar normalmente ou } \\
\text { cantar uma música enquanto se movimenta. }\end{array}$ & $\begin{array}{l}\text { São aquelas atividades que exigem } \\
\text { mínimo esforço físico e que causam } \\
\text { pequeno aumento da respiração e dos } \\
\text { batimentos cardíacos (abaixo de } 60 \% \text { da } \\
\text { FCmáx). O gasto energético é entre } 1,6 \\
\text { e } 2,9 \text { equivalentes metabólicos. Numa } \\
\text { escala de zero a } 10 \text {, a percepção de esforço } \\
\text { está entre } 3 \text { e } 4 . \text { Durante a realização de } \\
\text { atividade física leve, a pessoa consegue } \\
\text { respirar tranquilamente, conversar } \\
\text { normalmente ou mesmo cantar uma } \\
\text { música enquanto se movimenta. }\end{array}$ & $\begin{array}{l}\text { Leve: exige mínimo esforço físico e causa } \\
\text { pequeno aumento da respiração e dos seus } \\
\text { batimentos do coração. Numa escala de } \\
0 \text { a } 10 \text {, a percepção de esforço é de } 1 \text { a } 4 \text {. } \\
\text { Você vai conseguir respirar tranquilamente } \\
\text { e conversar normalmente enquanto se } \\
\text { movimenta ou até mesmo cantar uma } \\
\text { música. }\end{array}$ \\
\hline
\end{tabular}


Continuação do Quadro 3 - Processo de elaboração dos conceitos e terminologias das Intensidades da Atividade Física, para o Guia de Atividade Física para a População Brasileira 2020.

\begin{tabular}{|c|c|c|c|}
\hline Termos & $\begin{array}{l}\text { Proposição inicial do conceito } \\
\text { (Etapa 1) }\end{array}$ & $\begin{array}{l}\text { Proposição do conceito definido após } \\
\text { escuta aos GT } \\
\text { (Etapa 2) }\end{array}$ & $\begin{array}{l}\text { Conceito final definido após a consulta } \\
\text { pública } \\
\text { (Etapa 3) }\end{array}$ \\
\hline $\begin{array}{l}\text { Intensidade } \\
\text { moderada }\end{array}$ & $\begin{array}{l}\text { São aquelas que exigem esforço físico, que } \\
\text { fazem respirar mais forte que o normal e que } \\
\text { aumentam os batimentos cardíacos. O gasto } \\
\text { energético é entre } 3,0 \text { e } 5,9 \text { equivalentes } \\
\text { metabólicos. A percepção individual de } \\
\text { esforço está entre } 5 \text { e } 6 \text {, em uma escala de } \\
\text { zero a } 10 \text {. Nas atividades físicas moderadas, } \\
\text { consegue-se conversar com dificuldade } \\
\text { enquanto se movimenta, mas não consegue- } \\
\text { se cantar. }\end{array}$ & $\begin{array}{l}\text { São aquelas que exigem esforço físico, que } \\
\text { fazem respirar mais forte que o normal } \\
\text { e que aumentam moderadamente os } \\
\text { batimentos cardíacos (entre } 60 \% \text { a } 75 \% \\
\text { da FCmáx). O gasto energético é entre } \\
3,0 \text { e } 5,9 \text { equivalentes metabólicos. Numa } \\
\text { escala de zero a 10, a percepção de esforço } \\
\text { está entre } 5 \text { e } 6 \text {. Nas atividades físicas } \\
\text { moderadas, a pessoa consegue conversar } \\
\text { com dificuldade enquanto se movimenta, } \\
\text { mas não consegue cantar. }\end{array}$ & $\begin{array}{l}\text { Moderada: exige mais esforço físico, que } \\
\text { faz você respirar mais rápido que o normal } \\
\text { e que aumenta moderadamente os seus } \\
\text { batimentos do coração. Numa escala de } 0 \text { a } \\
10 \text {, a percepção de esforço é } 5 \text { e } 6 \text {. Você vai } \\
\text { conseguir conversar } \\
\text { com dificuldade enquanto se movimenta e } \\
\text { não vai conseguir cantar. }\end{array}$ \\
\hline $\begin{array}{l}\text { Intensidade } \\
\text { vigorosa }\end{array}$ & $\begin{array}{l}\text { São aquelas que exigem um grande esforço } \\
\text { físico, que fazem respirar muito mais forte } \\
\text { que o normal e que aumentam muito os } \\
\text { batimentos cardíacos. O gasto energético em } \\
\text { equivalentes metabólicos é maior que } 6,0 \text { em } \\
\text { adultos e maior que } 7,0 \text { em crianças e jovens. } \\
\text { A percepção individual de esforço está } \\
\text { entre } 7 \text { e } 8 \text {, em uma escala de zero a } 10 \text {. Nas } \\
\text { atividades físicas vigorosas, não se consegue } \\
\text { nem conversar com outra pessoa enquanto se } \\
\text { movimenta. }\end{array}$ & $\begin{array}{l}\text { São aquelas que exigem um grande } \\
\text { esforço físico, que fazem respirar muito } \\
\text { mais forte que o normal e que aumentam } \\
\text { muito os batimentos cardíacos (acima de } \\
75 \% \text { da FCmáx). O gasto energético é } \\
\text { maior que } 6,0 \text { equivalentes metabólicos. } \\
\text { Numa escala de zero a } 10 \text {, a percepção de } \\
\text { esforço está entre } 7 \text { e } 8 \text {. Nas atividades } \\
\text { físicas vigorosas, a pessoa não consegue } \\
\text { conversar enquanto se movimenta. }\end{array}$ & $\begin{array}{l}\text { Vigorosa: exige um grande esforço físico, } \\
\text { que faz você respirar muito mais rápido } \\
\text { que o normal e que aumenta muito os seus } \\
\text { batimentos do coração. Numa escala de } 0 \text { a } \\
10 \text {, a percepção de esforço é } 7 \text { e } 8 \text {. Você não } \\
\text { vai conseguir nem conversar enquanto se } \\
\text { movimenta. }\end{array}$ \\
\hline
\end{tabular}

\section{Atividade física}

A proposta do conceito da AF foi baseada em outros guias, especialmente o da $\mathrm{OMS}^{3}$. Em sua versão atualizada, a OMS definiu AF como "qualquer movimento corporal produzido pelos músculos esqueléticos que requeira gasto de energia" (p.vii) ${ }^{1}$. A partir desse conceito, realizamos os ajustes necessários para a realidade brasileira utilizando definições apresentadas em artigos ${ }^{9,12}$ e nos relatórios nacionais ${ }^{2,13}$. Desse modo, a definição do conceito de AF vem ampliando o seu escopo. Como exemplo, uma discussão sobre a definição do termo, sugere que "atividade física envolve pessoas se movendo, agindo e atuando em espaços e contextos culturalmente específicos e influenciados por uma gama única de interesses, emoçôes, ideias, instruçôes e relacionamentos".

Nesta mesma direção, uma carta na revista The Lancet destaca que a AF é um ato político, social e emocional complexo, e discute que recomendações de intensidade ou de tempo podem atrapalhar na tomada de decisões das pessoas ${ }^{19}$. Corroborando essas ideias, Silva et al. ${ }^{20}$ entendem a AF como parte da experiência de vida cotidiana. Nesta perspectiva, é necessário considerar que cada indivíduo e comunidade tem suas próprias preferências, que são influenciadas por símbolos, valores e significados. $\mathrm{E}$ ainda, de acordo com o relatório do PNUD, vivemos hoje um estágio de transição, no qual a AF era considerada simplesmente como "gasto energético expandido" para um novo modelo em que a AF é entendida como um comportamento ou prática multidimensional que envolve o corpo humano em movimento, e que possibilita a interação consigo, com o outro e com o meio, em um determinado contexto sociocultural ${ }^{13}$.

A partir das definições dos conceitos, ora com uma visão biológica ${ }^{1,12}$, e ora com uma visão holística ${ }^{9,13,19}$, o conceito produzido para o Guia avançou no sentido de apresentar possibilidades de prática de $\mathrm{AF}$ em um contexto social mais amplo, contemplando populações tão diversas e que vivem em contextos sociais diferenciados, como no Brasil. Em outros guias, publicados nos Estados Unidos ${ }^{5}$, na Espanha ${ }^{7}$ e na Irlanda ${ }^{8}$, a definição de AF é abordada a partir do conceito voltado ao movimento corporal produzido pela contração do músculo esquelético e que resulta em gasto energético. Desse modo, o Guia se diferencia dos guias de AF de outros países, mais centrados em uma visão biologicista e da sua relação com a saúde.

\section{Comportamento sedentário}

O conceito de CS foi amplamente aceito nas consultas realizadas e poucas sugestões foram indicadas. Isso pode ser em decorrência de termos considerado como base o conceito sugerido pela rede internacional $\mathrm{Se}$ dentary Behavior Research Network (SBRN) ${ }^{14}$. Recen- 
temente, a SBRN realizou um projeto de consenso de terminologia para padronizar o conceito de CS por meio de um processo sistemático de construção que incluiu revisão de literatura, definições e revisão dos termos-chave por um comitê diretor, desenvolvimento de um modelo consensual e análise e contribuição de especialistas ${ }^{21}$. No resultado desse processo, o termo CS foi conceituado como "qualquer comportamento realizado no periodo de vigília caracterizado por um gasto energético $\leq 1,5$ equivalentes metabólicos (METs), na posição sentada, reclinada ou deitada" ${ }^{2}$. Pensando na proposta desse conceito para o Guia, o GT Domínios propôs a tradução de "vigília" para "quando você está acordado" e da informação "gasto energético $\leq 1,5$ equivalentes metabólicos" para "gastando pouca energia”. Além disso, optou-se por repetir nos exemplos a informação "quando você está sentado, reclinado ou deitado" para que fique claro que o CS ocorre em todas essas posições.

\section{Domínios da AF}

Quanto aos domínios da AF, além do conceito, outras questões como nome dos domínios, termos a serem utilizados na definição conceitual e exemplos foram amplamente discutidos.

No processo de elaboração conceitual consideramos a importância de definir domínios da AF para a população brasileira, uma vez observada a inexistência de uma definição teórica e operacional na literatura nacional. Conforme a definição apresentada, os domínios representam o propósito da $\mathrm{AF}$, podendo ser realizada no tempo livre, no deslocamento, no trabalho ou estudo e nas tarefas domésticas. Apesar dessa definição ter sido suprimida da versão final do Guia, o GT Domínios considera este resultado importante, pois auxiliará e contribuirá para a produção do conhecimento, formação profissional e desenvolvimento de políticas públicas à promoção da $\mathrm{AF}$.

As apresentações dos quatro domínios não são frequentemente descritas em guias e recomendações, restringindo em sua maioria às estratégias de comunicação nos domínios de lazer e deslocamento. De fato, estes domínios possibilitam maior investimento das políticas públicas voltadas à promoção da AF. Além disso, parecem mais interessantes para promover a mudança de comportamento. Porém, ao se tratar de um contínuo diário, é importante pautar as diferentes oportunidades de se ter um estilo de vida ativo ${ }^{22}$. A OMS recentemente iniciou uma campanha mundial, chamada Let's be active, considerando que "todo movimento é impor- tante"23 e incluiu toda e qualquer oportunidade de ser ativo como algo importante para a saúde ${ }^{1}$.

A proposição dos conceitos dos domínios da AF pautou-se nas recomendações da OMS publicadas recentemente ${ }^{1}$. Vale mencionar que os nomes dos domínios da AF para o presente Guia foram amplamente discutidos: lazer ou tempo livre, transporte ou deslocamento, tarefas ou atividades domésticas, trabalho e atividades educacionais ou estudo formal ou escola. As tomadas de decisões em cada instância do processo de proposição dos conceitos objetivaram a tradução do termo estabelecido no cenário acadêmico para a população em geral, tendo grande influência em nossas decisões como os termos são compreendidos fora do ambiente acadêmico.

O nome do domínio do tempo livre apresentou divergências no GT Domínios ao longo do processo de proposição do conceito, especialmente diante dos estudos que o denominam como lazer ${ }^{24}$. A principal diferença estabelecida entre tempo livre e lazer é a possibilidade de escolha desinteressada de uma ação objetivando o divertimento, em detrimento do período de ócio ${ }^{24}$. $\mathrm{O}$ tempo livre necessariamente não é lazer, porém o lazer está inserido no tempo livre ou liberado ${ }^{25}$. Levando em consideração outros guias de AF, o domínio do lazer é mais presente, e sua nomenclatura pode ser condicionada à faixa etária da população alvo da recomendação (crianças, jovens, adultos ou idosos). Todavia, ainda existem variações atreladas à aspectos sociais, o que leva à reflexão da influência contextual e social do termo lazer e seu respectivo reconhecimento de oportunidade pela população em geral. O significado de lazer não é reconhecido pelos grupos vulneráveis da sociedade, em sua parte identificados como negros e imigrantes ${ }^{26}$.

Considerando os dados da escuta, o termo deslocamento para o nome do domínio foi identificado como mais claro e acessível à população brasileira em comparação ao termo transporte, amplamente utilizado na literatura internacional. Este domínio possui importante papel no processo de mudança de comportamento. Apesar da grande influência de fatores ambientais, a possibilidade e oportunidades em escolhas saudáveis de rotina ou esporádicas oferecem um grande potencial de promoção da $\mathrm{AF}$ por meio desse domínio ${ }^{27}$. Ao valorizá-lo, o GT objetiva informar e motivar a população para deslocar-se de forma ativa, assim como estimular gestores e profissionais para a viabilização de tais oportunidades de prática ao público.

Com relação ao domínio das AF realizadas no tra- 
balho, foi discutida a inclusão das atividades educacionais, considerando as inquietações do GT Domínios e as sugestões da escuta com pesquisadores. Após discussões com o CC do Guia, foi acrescido ao domínio do trabalho o termo estudo. Portanto, além da inclusão do termo estudo no nome do domínio, as atividades educacionais também foram contempladas nesse conceito. Diante do desafio de contemplar a população brasileira em todos os ciclos vitais, foi reconhecido que cada grupo etário possui compromisso com um espaço e atividade de contribuição formativa, social e/ou financeira. Desta maneira, os conceitos e os exemplos utilizados poderão contribuir para a identificação da população com oportunidades de AF, bem como importantes iniciativas organizacionais.

O domínio das tarefas domésticas foi considerado apenas na publicação do guia canadense ${ }^{6}$ e recentemente, nas recomendações de $\mathrm{AF}$ da $\mathrm{OMS}^{1}$. No Brasil, esse domínio ainda possui um caráter representativo em dois estratos da população: mulheres adultas e idosos. Visando a mensagem de que pequenas mudanças são importantes para indicadores de saúde, podemos considerar que as AF não estruturadas e planejadas, como as realizadas neste domínio, são de suma importância para a substituição de longos períodos em $\mathrm{CS}^{1,3}$.

\section{Intensidades da atividade física}

$\mathrm{O}$ que é considerada uma $\mathrm{AF}$ leve, moderada ou vigorosa? Com base nas pesquisas realizadas em guias internacionais ${ }^{3-5}$, verificamos a utilização de parâmetros fisiológicos, como a respiração, os batimentos cardíacos e o gasto energético. Mas, como traduzir esses termos para a população? Buscamos avançar no conceito de intensidade para além da "dose" do esforço físico, objetivando apresentar uma forma mais clara, tanto para profissionais quanto para a população em geral.

Além dos parâmetros fisiológicos, optamos em acrescentar a percepção do esforço, também utilizada em outros guias ${ }^{3,5}$. A avaliação do esforço percebido é uma medida psicofisiológica amplamente conhecida e utilizada nas áreas de AF, Educação Física, reabilitação e no campo científico ${ }^{28}$. Destacamos, que após as considerações do CC foi definida a expressão percepção do esforço a ser adotada no Guia.

Além disso, para simplificar o entendimento sobre a percepção do esforço físico em intensidade leve, moderada ou vigorosa, adotamos no conceito o teste da fala (Talk Test), utilizado por outros guias ${ }^{4,8}$. O pressuposto básico do teste da fala é que o indivíduo não consegue manter uma conversa confortável em determina- das intensidades e que essa inabilidade é associada a ventilação e outras respostas fisiológicas ${ }^{29}$. $\mathrm{O}$ teste da fala como uma medida de percepção de esforço ganhou aderência e atenção no contexto acadêmico na última década com pesquisas que objetivam evidências de sua validade e aplicação ${ }^{28,29}$.

Ademais, o guia irlândes ${ }^{8}$, por exemplo, apresenta no seu conceito de intensidade a sensação de calor e sudorese. Porém, após discussões nas reuniões do GT Domínios, optamos por não incluir esse tipo de informação, pois o Brasil, de forma geral, apresenta um clima tropical com altas temperaturas no verão, e a sensação de calor e sudorese poderia não refletir necessariamente esforços físicos.

\section{Exemplos}

Os exemplos sugeridos para os conceitos de AF e domínios da AF foram discutidos considerando os diferentes grupos etários, aspectos culturais e regionais e as AF mais praticadas pela população brasileira ${ }^{2,13}$. Buscou-se informar à população que uma mesma $\mathrm{AF}$, como o caminhar, pode ser realizada em diferentes domínios, e ampliar o que é comumente exemplificado como AF, considerando a diversidade cultural brasileira.

Especificamente no domínio das tarefas domésticas foram exploradas outras atividades além do tradicional "varrer e lavar", como aquelas voltadas ao cuidado com a família (dar banho em crianças ou trocar vestimenta de idosos que requerem cuidados), que são importantes e devem ser consideradas. Além disso, buscou-se ampliar os exemplos para além das atividades realizadas "dentro de casa" ou "no jardim ou quintal" (comumente avaliadas subjetivamente neste formato), valorizando outras atividades como "fazer compras" ao considerar a AF realizada dentro do estabelecimento comercial (supermercado, feira, farmácia, lojas, dentre outros). Finalmente, optou-se por não utilizar exemplos nas terminologias das intensidades, pois uma mesma $\mathrm{AF}$ pode ser desenvolvida em um continuum de intensidades. Como exemplo, limpar o chão ou caminhar ou correr podem ser realizados nas intensidades leve, moderada ou vigorosa dependendo de fatores individuais interoceptivos e exteroceptivos, como o exercício físico, a título de exemplo.

Acreditamos que os métodos utilizados no processo de avaliação desses conceitos forneçam subsídios para a utilização pela população. As discussões realizadas amadureceram os exemplos discutidos pelos pesquisadores, buscando fornecer à sociedade conceitos com termos e exemplos mais populares em prol dos mais 
técnicos, mais claros e de fácil entendimento.

Importante esclarecer que o Guia apresenta em seu conteúdo o conceito de exercício físico (EF), porém, sua inclusão ocorreu após a conclusão da avaliação de validade e clareza dos demais conceitos (etapa 2). Contudo, uma vez que o conceito de EF existente é aceito na literatura ${ }^{1,12,13}$, remetendo à sistematização da $\mathrm{AF}$, ele foi proposto pelo GT (etapa 1) e submetido à etapa 3 (consulta pública). A OMS ${ }^{1}$ e o $\mathrm{PNUD}^{13}$ destacam que o EF tem elementos em comum com a AF, caracterizando-o como uma subcategoria desta. Diante disso, todo EF é AF, mas nem toda AF é EF. O conceito de EF está contemplado nos guias internacionais e está alinhado com o objetivo de melhorar a saúde, a aptidão física ${ }^{5}$ a forma física ${ }^{8}$ e na manutenção de um ou mais componentes da aptidão física ${ }^{7}$. Portanto, esses conceitos se assemelham ao presente no Guia (melhorar ou manter as capacidades físicas), ao qual também foi acrescido o termo "peso adequado" com referência a um elemento da aptidão física relacionado à saúde.

O processo de avaliação da validade e clareza dos conceitos e terminologias do Guia apresenta pontos fortes e limitações. Como ponto forte, destacamos a composição do GT constituído por pesquisadores com atuações em subáreas da saúde pública (epidemiologia, políticas públicas) e saúde coletiva (saúde do idoso, saúde mental e saúde da criança e do adolescente); o processo sistematizado empregado para proposição dos conceitos do Guia; a participação de pesquisadores especialistas (etapa 2) e da população em geral (etapa 3), com representação de todas as regiões do país. Enumeramos como limitações a ausência de consulta às fontes bibliográficas que não foram publicadas em periódicos científicos indexados; o não desenvolvimento de estratégias de roda de conversa presenciais, em decorrência das medidas sanitárias do enfrentamento à pandemia da COVID-19; e a não realização da etapa 2 para o conceito de EF, devido a sua inclusão no Guia ocorrer após o término desta etapa.

A avaliação dos conceitos adotados no Guia considerou a necessidade de tornar claros e adequados os conceitos e terminologias existentes na literatura científica para a população, profissionais e gestores contribuindo para a tomada de decisões na adoção de um estilo de vida ativo e na elaboração de políticas de saúde voltadas à promoção da $\mathrm{AF}$. Conclui-se que os conceitos e terminologias propostos pelo GT Domínios para compor o Guia, após a avaliação de pesquisadores especialistas e da população consultada, são válidos e claros.

\section{Conflito de interesse}

Os autores declaram não haver conflito de interesses.

\section{Financiamento}

Este trabalho faz parte do Guia Brasileiro de Atividade Física, que foi financiado pelo Ministério da Saúde do Brasil por meio do Termo de Execução Descentralizada n ${ }^{\circ}$ 56/2019 (projeto: 79224219002/2019; processo: 25000.171034/2019-27)

\section{Contribuição dos autores}

Contribuição dos autores Benedetti TRB, participou da concepção dos conceitos e redação do manuscrito, e interpretação dos dados. Borges LJ, participou da concepção dos conceitos, da criação dos questionários para validade e clareza, organização da coleta, análise e interpretação dos dados, redação do manuscrito. Streit IA, participou da concepção dos conceitos, da organização dos questionários para validade e clareza, organização da coleta, análise e interpretação dos dados, redação do manuscrito. Garcia LMT, participou da discussão e aprovação dos conceitos, da redação e revisão crítica e aprovação do manuscrito. Manta SW, participou da discussão e aprovação dos conceitos e revisão crítica do conteúdo intelectual. Mendonça $\mathrm{G}$, participou da discussão e aprovação dos conceitos, da redação e revisão crítica do conteúdo intelectual. Binotto MA, participou da discussão e aprovação dos conceitos, da redação e revisão crítica e aprovação do manuscrito. Christofoletti M, participou da discussão e aprovação dos conceitos, da redação e revisão crítica e aprovação do manuscrito. Silva-Júnior FL participou da discussão e aprovação dos conceitos, da redação e revisão crítica e aprovação do manuscrito. Hallal PC participou da concepção, redação e revisão geral do manuscrito. Papini CB participou da concepção dos conceitos, da validação e correção dos questionários de validade e clareza, análise e interpretação dos dados, redação do manuscrito.

\section{Agradecimentos}

Os autores agradecem ao Ministério da Saúde pelo financiamento ao Guia de Atividade Física para a População Brasileira, aos pesquisadores de outros GT pela análise dos conceitos quanto à clareza e validade, a todos que enviaram suas contribuições na consulta pública e ao Joilson Meneguci pela criação da figura.

\section{Referências}

1. World Health Organization. Guidelines on Physical Activity and Sedentary Behaviour. 2020; Disponível em: < https://www. who.int/publications/i/item/9789240015128 > [2021 janeiro].

2. Brasil. Vigitel Brasil 2019: vigilância de fatores de risco e proteção para doenças crônicas por inquérito telefônico: estimativas sobre frequência e distribuição sociodemográfica de fatores de risco e proteção para doenças crônicas nas capitais dos 26 estados brasileiros e no Distrito Federal em 2019. 2020; Disponível em: <http://bvsms.saude.gov.br/bvs/publicacoes/vigitel_brasil_2019_vigilancia_fatores_risco.pdf > [2021 janeiro]. 
3. World Health Organization. Global recommendations on physical activity for health. 2010; Disponível em: < https://www. who.int/publications/i/item/9789241599979> [2021 janeiro].

4. Department of Health. UK Chief Medical Officer's physical activity guidelines. 2019; Disponível em: < https://assets. publishing.service.gov.uk/government/uploads/system/ uploads/attachment_data/file/832868/uk-chief-medicalofficers-physical-activity-guidelines.pdf> [2021 janeiro].

5. U.S. Department of Health and Human Services. Physical Activity Guidelines for Americans. Physical Activity Guidelines for Americans. 2018; Disponível em: < https:// health.gov/sites/default/files/2019-09/Physical_Activity_ Guidelines_2nd_edition.pdf> [2021 janeiro].

6. Canadian Society for Exercise Physiology; Canadian Physical Activity Guidelines. Canadian Sedentary Behaviour Guidelines. 2012; Disponível em: < https://ymca.ca/CWP/ media/YMCA-National/Documents/Our\%20Impact\%20 page\%20resources/CSEP_Guidelines_Handbook.pdf> [2021 janeiro].

7. Ministerio de Sanidad, Servicios Sociales e Igualdad. Actividad Física para la Salud y Reducción del Sedentarismo: Recomendaciones para la población. 2015. Disponível em: <https://www.mscbs.gob.es/profesionales/saludPublica/ prevPromocion/Estrategia/docs/Recomendaciones_ ActivFisica_para_la_Salud.pdf $>$ [2021 janeiro].

8. Department of Health and Children. The National Guidelines on Physical Activity for Ireland.2009; Disponível em: <https:// www.hse.ie/eng/about/who/healthwellbeing/our-priorityprogrammes/heal/heal-docs/the-national-guidelines-onphysical-activity-for-ireland.pdf> [2021 janeiro].

9. Piggin J. What is physical activity? A holistic definition for teachers, researchers and policy makers. Front Sports Act Living. 2020; 2(72):e-2-7.

10. Haskell WL, Lee I, Pate RR, Powell KE, Blair SN, Franklin $\mathrm{BA}$, et al. Physical activity and public health: updated recommendation for adults from the American College of Sports Medicine and the American Heart Association. Med Sci Sports Exerc. 2007;39(8):1423-34.

11. Ainsworth BE, Haskell WL, Whitt MC, Irwin ML, Swartz AM, Strath SJ, et al. Compendium of physical activities: an update of activity codes and MET intensities. Med Sci Sports Exerc. 2000;32(Supp19):S498-516.

12. Caspersen CJ, Powell KE, Christenson GM. Physical-activity, exercise, and physical-fitness - definitions and distinctions for health-related research. Public Health Rep.1985;100(2):126-31.

13. Brasil. Relatório de Desenvolvimento Humano Nacional - Movimento é Vida: Atividades Físicas e Esportivas para Todas as Pessoas: 2017. Brasília: PNUD; 2017.

14. Sedentary Behaviour Research Network. Sedentary Behavior Research Network. Terminology Consensus Project. 2017; Disponível em: <https://www.sedentarybehaviour.org/sbrnterminology-consensus-project/\#consensus-definitions [2021 janeiro].

15. Santos SG, Schutz GR, Sant'ana ASS, Farias ES, Fares D, Minatto $\mathrm{G}$, et al. Métodos e técnicas de pesquisa quantitativa aplicada à educação física. 1. ed. Florianópolis: Tribo da Ilha, 2011. 237p.
16. Thomas JR, Nelson JK, Silverman SJ. Métodos de pesquisa em atividade física. 5 ed. Porto Alegre: Artmed, 2007

17. INVOLVE. Public involvement in research and research ethics committee review. 2016. Disponível em: <https:// www.invo.org.uk/wp-content/uploads/2016/05/HRAINVOLVE-updated-statement-2016.pdf>

18. Brasil. Resolução no 510, de 7 de abril de 2016. Ministério da Saúde. Conselho Nacional de Saúde. 2016.

19. Matias TS, Piggin J. Physical activity promotion: can a focus on disease limit successful messaging? Lancet. 2020; 8(10):e1263.

20. Silva KS, Garcia LMT, Rabacow FM, Rezende LFM, Sá TH. Physical activity as part of daily living: Moving beyond quantitative recommendations. Prev Med. 2017; 96:160-62.

21. Tremblay MS, Aubert S, Barnes JD, Saunders TJ, Carson V, Latimer-Cheung AE, et al. Sedentary Behavior Research Network (SBRN) - Terminology Consensus Project process and outcome. Int. J. Behav. Nutr Phys Act. 2017;14(75):e2-17.

22. Ross R, Chaput JP, Giangregorio LM, Janssen I, Saunders TJ, Kho M E et al. Canadian 24-Hour Movement Guidelines for Adults aged 18-64 years and Adults aged 65 years or older: an integration of physical activity, sedentary behaviour, and sleep. Appl Physiol Nutr Metab, 2020; 45(10): S57-S102.

23. World Health Organization. Global action plan on physical activity 2018-2030: more active people for a healthier world. 2018; Disponível em: https://apps.who.int/iris/bitstream/ha ndle/10665/272722/9789241514187-eng.pdf?ua=1> [2021 janeiro].

24. Marcellino NC. Lúdico, educação e educação física. Ijuí: 4 Ed. Unijuí, 2013.

25. Cavallari VR, Zacharias V. Trabalhando com recreação. 2. ed. São Paulo: Ícone, 1995.

26. Stodolska M. Research on Race, Ethnicity, Immigration, and Leisure: Have We Missed the Boat? Leis Sci 2018;40(12):43-53.

27. Hilland TA, Bourke M, Wiesner G, Garcia EB, Parker AG, Pascoe M, et al. Correlates of walking among disadvantaged groups: A systematic review. Health Place. 2020;63:102337.

28. Foster C, Porcari JP, Anderson J, Paulson M, Smaczny D, Webber $\mathrm{H}$, et al. The talk test as a marker of exercise training intensity. J Cardiopulm Rehabil Prev. 2008;28(1):24-30.

29. Quinn TJ, Coons BA. The Talk Test and its relationship with the ventilatory and lactate thresholds. J Sports Sci, 2011;29(11):1175-82.

30. Marques, JBV, Freitas, D. Método DELPHI: caracterização e potencialidades na pesquisa em Educação. Pro-Posições 29.2 (2018):389-415. 